\title{
Proof of principle of Electron Cyclotron Emission as a complementary diagnostic for the plasma position
}

\author{
C.J. Rapson*, S. Denk*, R. Fischer*, L. Giannone*, A. Gude*, W. Treutterer*, M. Willensdorfer* and the ASDEX Upgrade \\ * Max Planck Institute for Plasma Physics, Boltzmannstr. 2, 85748 Garching, Germany \\ Email: chris.rapson@ipp.mpg.de
}

\begin{abstract}
Today's tokamaks use magnetic diagnostics to control the plasma position. In future fusion reactors (e.g. DEMO) the performance of magnetic diagnostics will be compromised by integrator drifts over long pulses and by radiation. Microwave diagnostics are good alternatives, as they are expected to be robust to the harsh radiation environment. Reflectometry has shown its potential to measure the density profile and hence to derive the separatrix position. This submission demonstrates how Electron Cyclotron Emission (ECE) can do the same via the electron temperature $\left(T_{e}\right)$ profile.

It will be shown that $T_{e}$ at the separatrix can be calculated as a function of several well-diagnosed plasma parameters for a wide range of scenarios. From ECE measurements, the $T_{e}$ profile can be derived, taking into account problematic effects such as shinethrough and corrupt measurement channels. Trivially, finding the position where the profile matches the separatrix temperature gives the separatrix position.

In a proof-of-principle experiment on ASDEX Upgrade, the radial plasma position was controlled using the separatrix position derived from ECE measurements. After initial success, problems with cutoff and latency led to a loss of control. An outline is given on how to remedy these issues and develop this proop-of-principle into a viable diagnostic for fusion reactors.
\end{abstract}

\section{INTRODUCTION}

If DEMO reaches its target of producing significant fusion power, then it will unavoidably produce high levels of neutron radiation. The radiation presents a challenge to many conventional diagnostics, in particular magnetic coils [1]-[3] which are typically the main diagnostic used for plasma position control. Coils can be shielded, but then their dynamic response becomes slow. Magnetic diagnostics are also subject to drift, so will need correcting over long pulses [4]. Developing accurate and reliable diagnostics is extremely important since position control actuators in DEMO are challenged even in simulations with ideal diagnostics [5] and loss of control would lead to a disruption, which must be avoided at all costs.

One option is to use radiation-hard Hall probes, which can be placed close to the plasma [6]. Another is reflectometry, which estimates the separatrix position based on the density profile [7]. This paper presents a new possibility; using Electron Cyclotron Emission (ECE) to measure the electron temperature $\left(T_{e}\right)$ profile and estimate the separatrix position, analagous to reflectometry. Since ECE, like reflectometry, uses microwaves, it belongs to the small group of diagnostics which are expected to be feasible in the DEMO environment [1].

\section{METHOD}

To convert raw ECE measurements into a $T_{e}$ profile, artefacts such as corrupt channels and shinethrough [8] must be detected and removed. Artefacts from cutoff are more complicated and not treated at this stage. Channels are marked as corrupt if the measurement is outside the range $-10<T_{e}<$ $10^{5} \mathrm{eV}$ or if $T_{e}$ is more-than-double/less-than-half or more than $100 \mathrm{eV}$ higher/lower than both neighbouring channels. Channels with radial position $\mathrm{R}<1.85 \mathrm{~m}$ are ignored since they are far away from the separatrix. The shinethrough is detected as a local minimum with $T_{e}<1 \mathrm{keV}$, where the depth of the dip is at least $100 \mathrm{eV}$. Measurements from a radial location outside this minimum are ignored. Finally, a spatial low pass filter is used to smooth the profile. A comparison of raw data and the sanitised profile can be seen in fig 1 .

Analysing data from a large number of shots, it was found that the temperature at the separatrix $T_{e, s e p}$ could be well described by a function of the stored energy $\left(W_{M H D}\right)$, heating power $\left(P_{N B I}+P_{I C}+P_{E C}+P_{o h m}=P_{t o t}\right)$, edge density $\left(n_{e, e d g e}\right)$ and radiating impurities $\left(N_{2}\right)$ :

$$
\begin{gathered}
T_{e, s e p}=26+4 e^{-4} W_{M H D}+8 e^{-8}\left(\log _{10}\left(W_{M H D}^{\frac{1}{4}}\right)\right)^{50}+100 \frac{P_{N B I}}{P_{t o t}} \\
-80\left(\frac{P_{N B I}}{P_{t o t}}\right)^{2}+10 \frac{P_{E C}}{P_{t o t}}+50\left(\frac{P_{E C}}{P_{t o t}}\right)^{2}-200 \frac{P_{I C}}{P_{t o t}} \\
+300\left(\frac{P_{I C}}{P_{t o t}}\right)^{2}-1 e^{-5} P_{t o t}-4 e^{-19} n_{e, e d g e}-1 e^{-2} N_{2}
\end{gathered}
$$

Coefficients were fitted after removing outliers caused by pellets, impurity events, cutoff or fast transients such as beam blips. Other parameters such as plasma current, toroidal magnetic field, and core radiation were found to have an insignificant effect. The effect of error fields from magnetic perturbation coils was observed but not yet included in the fit.

To demonstrate the validity of the method, fig. 2 compares the estimate of the separatrix position $R_{s e p, E C E}$ with the magnetic separatrix $R_{s e p, m a g}$ for two very different scenarios. Both the absolute values and the dynamics match - even down to the resolution of individual ELMs. Since $R_{s e p, E C E}$ has larger high-frequency components than $R_{s e p, m a g}$, a temporal low pass filter was added. Note that equation (1) performs much better than the other two methods for estimating $T_{e, s e p}$. 


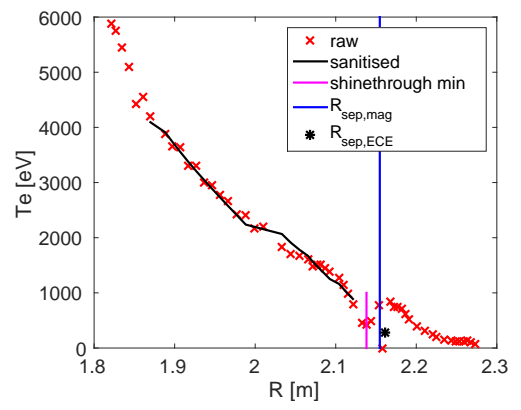

Fig. 1. A sanitised profile (-) is extracted from raw ECE data (x). Note the corrupt channel measuring $0 \mathrm{eV}$ and the unrealistically high Te measured around $\mathrm{R}=2.17 \mathrm{~m}$ due to shinethrough.
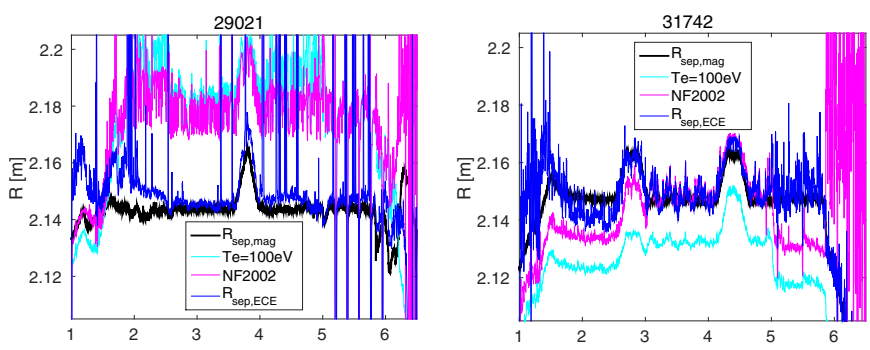

Fig. 2. Comparison of $R_{s e p, E C E}$ with $R_{s e p, m a g}$. Also plotted are a naive estimate using $T_{e, s e p}=100 \mathrm{eV}$ and a simple model for SOL transport given in ref. [9]. Pellets are launched in $\# 29021$ from $t=5 \mathrm{~s}$ onwards, and the gas supply fluctuates with $4 \mathrm{~Hz}$ in \#31742.

\section{EXPERIMENTAL RESULTS}

Due to unscheduled changes at the end of the AUG campaign, the experiment was run in an upper single null (USN) configuration, and during the changeover to hydrogen gas. The routine was shown to be surprisingly robust to running in USN, however uncertainty in the amount of hydrogen to puff led to higher-than-expected density and the ECE was in cutoff from $\mathrm{t}=2.3 \mathrm{~s}$. As noted before, the routine does not detect cutoff, so from this point on $R_{s e p, E C E}$ deviated from $R_{s e p, m a g}$ until the discharge disrupted, as shown in fig. 3. Until $\mathrm{t}=2.3 \mathrm{~s}$ however, there is much that can be learned. The transition from the magnetic to the ECE controller at $\mathrm{t}=1.8 \mathrm{~s}$ was successful, and a disturbance shortly after was rejected. Oscillations appear from $\mathrm{t}=2.1 \mathrm{~s}$ that may be due to the high density, or to the latency of the ECE measurements.

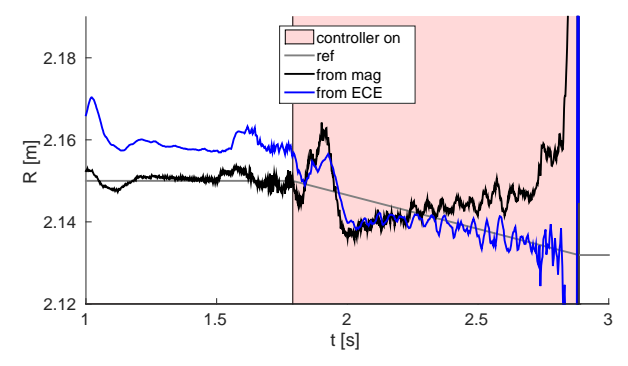

Fig. 3. ASDEX Upgrade shot using $R_{\text {sep, mag }}$ to control the radial plasma position until $\mathrm{t}=1.8 \mathrm{~s}$, and then switching to $R_{s e p, E C E}$. The reference trajectory is shown in grey.

\section{Future WORK}

A trivial improvement will be to reduce the latency from $6 \mathrm{~ms}$ to $2 \mathrm{~ms}$ for real-time ECE measurements. This upgrade is already planned for sawtooth control experiments. It would be advantageous to use an observer with a forward model and a transport model to estimate the $T_{e}$ profile. The forward model will be especially critical in DEMO [10]. Cutoff should be detected, perhaps using ray-tracing. Indirect dependencies on magnetic measurements $\left(W_{M H D}\right.$ and $\left.n_{e, e d g e}\right)$ should be assessed to see if non-magnetic replacements can be found.

So far, only $R_{\text {sep }}$ has been considered, but it is equally possible to detect and control the vertical plasma position with ECE. If one assumes that the core is up-down symmetric, then an array of ECE lines of sight can localise the plasma centre. In AUG, this could be emulated using a soft X-ray camera.

Looking towards the future, the empirical function used here to calculate $T_{e, s e p}$ should be reformulated from physics principles, such that it can be extrapolated to DEMO. The feasibility of this method in a pellet fuelled scenario should be assessed, since DEMO forsees pellet fuelling. Finally, a Bayesian framework should be developed to combine estimates of the plasma position from all DEMO diagnostics conventional magnetic coils, Hall probes, reflectometry, ECE and any new concepts that may arise between now and then.

\section{ACKNOWLEDGMENT}

This work has been carried out within the framework of the EUROfusion Consortium and has received funding from the Euratom research and training programme 2014-2018 under grant agreement No 633053. The views and opinions expressed herein do not necessarily reflect those of the European Commission

\section{REFERENCES}

[1] W. Biel et al., "DEMO diagnostics and burn control," Fusion Eng. Des., vol. 96 - 97, pp. 8 - 15, 2015, proceedings of the 28th Symposium On Fusion Technology (SOFT-28). [Online]. Available: http://www.sciencedirect.com/science/article/pii/S0920379615000733

[2] A. Donné et al., "Diagnostics for plasma control on DEMO: challenges of implementation," Nucl. Fusion, vol. 52, no. 7, p. 074015, 2012. [Online]. Available: http://stacks.iop.org/0029-5515/52/i=7/a=074015

[3] G. Vayakis et al., "Generic diagnostic issues for a burning plasma experiment," Fusion Sci. Technol., vol. 53, no. 2, pp. 699-750, FEB 2008.

[4] R. Chavan et al., "The magnetics diagnostic set for ITER," Fusion Eng. Des., vol. 84, no. 2-6, pp. 295 - 299, 2009. [Online]. Available: http://www.sciencedirect.com/science/article/pii/S0920379609000052

[5] R. Albanese et al., "Magnetic control of DEMO plasmas," in 2nd IAEA DEMO Programme Workshop, Vienna, 17-20 December, 2013.

[6] I. Duran et al., "Recent results and challenges in development of metallic Hall sensors for fusion reactors," AIP Conference Proceedings, vol. 1612, pp. 31-34, 2014. [Online]. Available: http://scitation.aip.org/content/aip/proceeding/aipcp/10.1063/1.4894020

[7] J. Santos et al., "Enhancement of the ASDEX Upgrade real-time plasma position reflectometry diagnostic," Nuclear Science, IEEE Transactions on, vol. 62, no. 3, pp. 1195-1200, June 2015.

[8] S. K. Rathgeber et al., "Estimation of edge electron temperature profiles via forward modelling of the electron cyclotron radiation transport at ASDEX Upgrade," Plasma Phys. Contr. Fusion, vol. 55, no. 2, FEB 2013.

[9] A. Kallenbach et al., "Scaling of the pedestal density in type-I ELMy $\mathrm{H}$-mode discharges and the impact of upper and lower triangularity in JET and ASDEX Upgrade," Nucl. Fusion, vol. 42, no. 10, p. 1184, 2002. [Online]. Available: http://stacks.iop.org/0029-5515/42/i=10/a=302

[10] C. Sozzi et al., "Towards the detection of magnetohydrodynamics instabilities in a fusion reactor," AIP Conference Proceedings, vol. 1612, pp. 35-38, 2014. [Online]. Available: http://scitation.aip.org/ content/aip/proceeding/aipcp/10.1063/1.4894021 